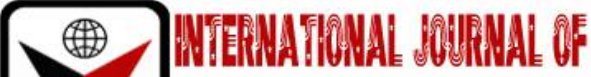

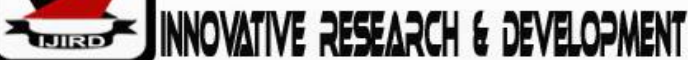

ISSN 2278 - 0211 (Online)

\section{A Comparison of Methods of Estimating the Parameters of the Three-Parameter Weibull Distribution with Application to Reliability Analysis}

\begin{tabular}{c}
\hline Ogbonna Uchenna Austine \\
Student, Department of Statistics, \\
Nnamdi Azikiwe University Awka, Anambra State, Nigeria \\
Obiora-Ilouno Happiness \\
Associate Professor, Department of Statistics, \\
Nnamdi Azikiwe University Awka, Anambra State, Nigeria
\end{tabular}

\begin{abstract}
:
In this work, eight methods of estimating the parameters of a three-parameter Weibull distribution were discussed and compared. The methods are; method of moments (MOM), maximum likelihood method (MLE), percentile method (PM), method of L-moments (LM), Teimouri and Gupta Beta (MGB), maximum product of spacing method (MPS), modified method of moments (MMM) and the Goda's polynomial method (GPM). A simulation study was carried out were samples of different sizes with different shape parameter $(\beta)$ values were generated and the five methods were applied to the samples. The root means square error (RMSE) was the basis for comparison of the methods based on their ability to estimate each parameter accurately. The Euclidean norm was also used to compare performance of methods based on their ability to accurately estimate the three parameters. The results show that the Mahdi and Gupta method is the best method for estimating the parameters of a three-parameter Weibull distribution in almost all the simulation conditions. The maximum product of spacing performed second best. It was also discovered that sample size does not really affect the choice of method but the accuracy of all the methods increases with sample. An application of these methods to real life data was also demonstrated here.
\end{abstract}

Keywords: Weibull distribution, estimation, simulation, comparison, L-moments

\section{Introduction}

Weibull distribution has proven to be a successful model for many product failure mechanisms because it is a flexible distribution given that it can for example take the form of either the exponential distribution or the appropriate normal distribution and can be skewed either positively or negatively. It is a distribution with a wide variety of possible failure rate curves. However, Lloyd (1967), ku et al (1972), Mc cool (1998) and Hammit (2004) as well as so many others have expanded the scope and usefulness of the Weibull distribution to other branches of statistics such as quality control. The Weibull distribution has the following pdf;

$$
f(x)=\frac{\beta}{\theta}\left(\frac{x-\gamma}{\theta}\right)^{\beta-1} e^{\left(\frac{x-\gamma}{\theta}\right)^{\beta}} x>\gamma, \beta>0, \theta>0
$$

The Weibull distribution has three parameters; the shape parameter $(\beta)$, the scale parameter $(\theta)$ and the location parameter $(\gamma)$.

The Maximum Likelihood Estimator is not available in closed form for two of the parameters of a three parameter Weibull distribution, therefore raising the need to review and compare alternative methods. In this paper, we review and compare eight methods of estimating the parameters of the three parameter-Weibull distribution.

The aim of this paper is to find the best method for estimating the parameters of the three-parameter Weibull distribution under different conditions.

\section{Review of Methods}

\subsection{Maximum Likelihood Method}

The maximum likelihood estimates are gotten by maximizing the log likelihood function of the distribution. The log-likelihood function of the three-parameter Weibull distribution which can be gotten from the pdf (equation 1.1) as; 


$$
\boldsymbol{L}\left(x_{1}, x_{2}, \ldots, x_{n}, \beta, \theta, \gamma\right)=n(\log \beta-\beta \log \theta)+(\beta-1) \sum_{i=1}^{n} \log \left(x_{i}-\gamma\right)-\frac{1}{\theta^{\beta}} \sum_{i=1}^{n} \log \left(x_{i}-\gamma\right)^{\beta}
$$

The estimates of $\beta, \theta$ and $\gamma$ are gotten by maximizing the equation above. This is done by differentiating the equations with respect to $\beta, \theta$ and $\gamma$ respectively, and equating the resulting expressions to zero.

The resulting equations are;

Differentiating equation 3.0 with respect to $\beta$, $\theta$ and $\gamma$ respectively gives;

$\frac{n}{\beta}+\sum_{i=1}^{n} \log \left(\frac{x_{i}-\gamma}{\theta}\right)-\sum_{i=1}^{n}\left(\frac{x_{i}-\gamma}{\theta}\right)^{\beta} \log \left(\frac{x_{i}-\gamma}{\theta}\right)=0$

$-\frac{n \beta}{\theta}+\frac{\beta}{\theta} \sum_{i=1}^{n}\left(\frac{x_{i}-\gamma}{\theta}\right)^{\beta}=0$

$-(\beta-1) \sum_{i=1}^{n} \frac{1}{x_{i}-\gamma}+\frac{\beta}{\theta} \sum_{i=1}^{n}\left(\frac{x_{i}-\gamma}{\theta}\right)^{\beta-1}=0$

The shape parameter cannot be isolated from any of these equations, which means the maximum likelihood estimate for the shape parameter cannot be expressed in closed form. The estimate for the shape parameter is gotten by solving these equations iteratively using Newton Raphson method.

From equation 3.7, the scale parameter is estimated using the following expression;

$\hat{\theta}=\left(\frac{\sum\left(x_{i}-\hat{\gamma}\right)^{\widehat{\beta}}}{n}\right)^{1 / \widehat{\beta}}$

The MLE for the location is given by;

$\hat{\gamma}=x_{(1)}=\min \left\{x_{1}, x_{2}, \ldots, x_{n}\right\}$

\subsection{Method of Moments}

The first three central moment are gotten from equations are given by;

$\mu_{1}=\gamma+\theta \Gamma\left(1+\frac{1}{\beta}\right)$

$\mu_{2}=\theta^{2}\left[\Gamma\left(1+\frac{2}{\beta}\right)-\Gamma^{2}\left(1+\frac{1}{\beta}\right)\right]$

$\mu_{3}=3 \Gamma\left(1+\frac{3}{\beta}\right)-3 \Gamma\left(1+\frac{1}{\beta}\right) \Gamma\left(1+\frac{2}{\beta}\right)+2 \Gamma^{3}\left(1+\frac{1}{\beta}\right)$

The coefficient of skewness is used to find an estimator for $\beta$. The coefficient of skewness is given by the expression;

$S_{k}=\sqrt{\frac{\mu_{3}^{2}}{\mu_{2}^{3}}}=\sqrt{\frac{\left[\Gamma\left(1+\frac{3}{\beta}\right)-3 \Gamma\left(1+\frac{1}{\beta}\right) \Gamma\left(1+\frac{2}{\beta}\right)+2 \Gamma^{3}\left(1+\frac{1}{\beta}\right)\right]^{2}}{\left[\Gamma\left(1+\frac{2}{\beta}\right)-\Gamma^{2}\left(1+\frac{1}{\beta}\right)\right]^{3}}}$

From equation 2.6 and 2.7 the estimators for $\theta$ and $\gamma$ are given by;

$$
\hat{\theta}=\sqrt{\frac{\mu_{2}}{\Gamma\left(1+\frac{2}{\hat{\beta}}\right)-\Gamma^{2}\left(1+\frac{1}{\hat{\beta}}\right)}} \text { and } \hat{\gamma}=\mu_{1}-\hat{\theta} \Gamma\left(1+\frac{1}{\beta}\right)
$$

\subsection{Percentile Method}

As the name implies, this method involves the use of quantiles. This method was derived by Dubey (1967). He proposed an estimator based on the $17^{\text {th }}$ and $97^{\text {th }}$ percentiles for the shape parameter and $45^{\text {th }}$ and $82^{\text {nd }}$ percentiles for the scale parameter.

Let $P_{1}=0.167$ and $P_{2}=0.9737$

And define $K_{1}=\log \left(-\log \left(1-P_{1}\right)\right)-\log \left(-\log \left(1-P_{2}\right)\right)$

Let $Y_{1}$ and $Y_{2}$ represent the $100\left(P_{i}\right.$ th $)$ percentile from a sample given sample, then,

$\hat{\beta}=\frac{-K_{1}}{\log \left(Y_{1}\right)-\log \left(Y_{2}\right)}(2.11)$

To estimate the scale parameter, 
Let $P_{3}=0.3978$ and $P_{4}=0.8211$

Define $K_{2}=\log \left(-\log \left(1-P_{1}\right)\right)-\log \left(-\log \left(1-P_{2}\right)\right)$ and $K_{3}=-\log \left(1-P_{3}\right)$

Also define $w=1-\frac{\log \left(K_{3}\right)}{K_{2}}$

Then, $\hat{\theta}=e^{\left(w \log \left(Y_{3}\right)+(1-w) \log \left(Y_{4}\right)\right)}$

The location parameter is estimated using;

$\hat{\gamma}=100(0.1)$ th percentile

\subsection{Teimouriand Gupta Beta}

Teimouri and Gupta (2013) gave a useful theorem for constructing a simple, consistent and closed form estimator for $\beta$. And interestingly this estimator is independent of $\theta$. The theorem states; suppose $x_{1}, x_{2}, \ldots, x_{n}$ is a random sample from a Weibull distribution. Let $\rho$ denote the sample correlation coefficient between $x_{i}$ and their ranks. Let $\mathrm{C}$ and $\mathrm{S}$ denote the sample coefficient of variation and the sample standard deviation respectively. Then,

$\sigma_{x}=$ standard deviation $(x)$

Based on a corollary of this theorem they stated $\hat{\beta}=\frac{-\ln 2}{\ln \left[1-\frac{\rho}{\sqrt{3}}\left(\frac{1}{C}-\frac{\gamma}{S}\right)^{-1} \sqrt{\frac{n+1}{n-1}}\right]}$

Where

$\mu_{x}=E(x)$

and

An estimator for $\gamma$ is $x_{(1)}-\frac{1}{n}$ (Sirvanci and yang 1954).

$\therefore \hat{\beta}=\frac{-\ln 2}{\ln \left[1-\frac{\rho}{\sqrt{3}}\left(\frac{1}{C}-\frac{x_{(1)}-\frac{1}{n}}{S}\right)^{-1} \sqrt{\frac{n+1}{n-1}}\right]}$

This estimator is now independent of $\gamma$ and $\theta$.For this method, the maximum likelihood estimator for the scale parameter will be used;

$\hat{\theta}=\left(\frac{\sum\left(x_{i}-\gamma\right)^{\beta}}{n}\right)^{1 / \beta}(3.16)$ Where $\beta=\hat{\beta}$ and $\gamma=\hat{\gamma}$

\subsection{Method of L-Moments}

This method is based on quantiles and order statistics.

If $\mathrm{X}$ is a random variable having a distribution with distribution function $\mathrm{F}(\mathrm{x})$ and quantile function $\mathrm{x}(\mathrm{F})$ and let $x_{1}, x_{2}, \ldots, x_{n}$ be a random sample of size $\mathrm{n}$ from this distribution. Then $X_{1: n} \leq X_{2: n} \leq \cdots \leq X_{n: n}$ are the order statistic of the random sample which comes from the distribution of the random variable $X$. Let $X$ be a real valued random variable with cumulative distribution $F(x)$ and quantile function $x(F)$. For the Weibull distribution $x(F)=\theta[-\ln (1-F(x))]^{1 / \beta}+$ $\gamma$ (which is gotten by making $\mathrm{x}$ the subject of formula in the distribution function of the Weibull distribution). The expectation of an order statistic is given by;

The first three L-moments can be expressed as;

$\alpha_{1}=\gamma+\theta \Gamma\left(\frac{1}{\beta}+1\right)=E(x)$

$\alpha_{2}=\theta \Gamma\left(\frac{1}{\beta}+1\right)\left[1-\frac{1}{2^{\frac{1}{\beta}}}\right]$

$\alpha_{3}=\theta \Gamma\left(\frac{1}{\beta}+1\right)\left[1-\frac{3}{2^{\frac{1}{\beta}}}+\frac{2}{3^{\frac{1}{\beta}}}\right]$

Equating these to the sample L-moments say $\pi_{i}$ and solving equations 2.16, 2.17 and 2.18 does not yield explicit results for the estimators of parameters. However, Goda et al (2010) provided a solution which is to use the L-skewness to find the estimator of $\beta$. The coefficient of L-skewness is given by $\tau_{3}=\frac{\widehat{\alpha_{3}}}{\widehat{\alpha_{2}}}=\frac{\pi_{3}}{\pi_{2}}$

$$
\tau_{3}=\frac{\theta \Gamma\left(\frac{1}{\beta}+1\right)\left(1-\frac{3}{2^{1 / \beta}}+\frac{2}{3^{1 / \beta}}\right)}{\theta \Gamma\left(\frac{1}{\beta}+1\right)\left(1-\frac{1}{2^{1 / \beta}}\right)}=\frac{\left(1-\frac{3}{2^{1 / \beta}}+\frac{2}{3^{1 / \beta}}\right)}{\left(1-\frac{1}{2^{1 / \beta}}\right)}
$$

This equation is used to estimate $\beta$. The estimators for the other two parameters are gotten from equation 3.17 and 3.18 as; $\hat{\theta}=\frac{\pi_{2}}{\Gamma\left(\frac{1}{\beta}+1\right)\left(1-\frac{1}{2}\right)}$ and $\hat{\gamma}=\pi_{1}-\hat{\theta} \Gamma\left(\frac{1}{\beta}+1\right)$ 


\subsection{Goda's Polynomial}

Goda et al (2010) fitted a polynomial function to equation 2.19 and got;

$\hat{\beta}=285.3 \pi_{3}{ }^{6}-658.6 \pi_{3}{ }^{5}+622.8 \pi_{3}{ }^{4}-317.2 \pi_{3}{ }^{3}+98.52 \pi_{3}{ }^{2}-21.256 \pi_{3}+3.516$

This polynomial was fit with an error of 0.3 for $0.6<\beta<6$.

\subsection{Maximum Product of spacing}

The MPS method was introduced independently by Cheng and Amin (1983) and Ranneby (1984). The MPS criterion amounts to turning the parameters so that the product of probabilities of a new observation falling between each two neighboring sampling points in the set of order statistics is maximized. The sum of this probability is one.

Given an iid random sample $\left(x_{1}, x_{2}, \ldots, x_{n}\right)$ of size $\mathrm{n}$ from a univariate distribution $F\left(x, \theta_{0}\right)$ where $\theta_{0}$ is an unknown parameter to be estimated.

Let $\left(x_{(1)}, x_{(2)}, \ldots, x_{(n)}\right)$ be corresponding ordered sample that is the result of sorting of all observations from the smallest to the largest. Also, for convenience, let $x_{(0)}=-\infty$ and $x_{(n+1)}=+\infty$

The spacing $D_{i}$ are defined as the gaps between the values of the distribution function at adjacent ordered points Say, $D_{i}=F\left(x_{(i)}, \theta_{0}\right)-F\left(x_{(i-1)}, \theta_{0}\right) i=1,2, \ldots, n+1$

Then the maximum spacing estimates of $\theta_{0}$ is defined as a value that maximizes the logarithm of the geometric mean of the sample spacing.

$\max S_{n}\left(\theta_{0}\right)=\ln \left(\sqrt[n+1]{\prod_{i=1}^{n+1} D_{i}}\right)$

$$
=\ln \left(\prod_{i=1}^{n+1} D_{i}\right)^{1 / n+1}=\frac{1}{n+1} \ln \left(\prod_{i=1}^{n+1} D_{i}\right)
$$

$=\frac{1}{n+1} \sum_{i=1}^{n+1} \ln D_{i}=\frac{1}{n+1} \sum_{i=1}^{n+1}\left(F\left(x_{(i)}, \theta_{0}\right)-F\left(x_{(i-1)}, \theta_{0}\right)\right)$

Forthe Weibull distribution we have, $=\frac{1}{n+1} \sum_{i=1}^{n+1}\left(\left(1-e^{-\left(\frac{x_{i}-\gamma}{\theta}\right)^{\beta}}\right)-\left(1-e^{-\left(\frac{x_{i-1}-\gamma}{\theta}\right)^{\beta}}\right)\right)$

The estimators $(\beta, \theta$ and $\gamma)$ are gotten by maximizing the above equation. Since this equation is not differentiable, we used the Newton Raphson method for maximization.

\subsection{The Modified Method of Moments}

This modification of the method of moment was introduced by Cohen and Whitten (1982).

The modification is based on the assumption that; $\boldsymbol{E}\left[\boldsymbol{F}\left(\boldsymbol{x}_{\boldsymbol{r}}\right)\right]=\boldsymbol{F}\left(\boldsymbol{x}_{\boldsymbol{r}}\right)$.

Where $E($.$) is the usual expectation, x_{r}$ is the $r^{\text {th }}$ order statistic in a random sample of size $\mathrm{n}$ and $F\left(x_{r}\right)$ is the associated value of the cumulative distribution.

Cohen and Whitten also stated that $\boldsymbol{E}\left[\boldsymbol{F}\left(\boldsymbol{x}_{\boldsymbol{r}}\right)\right]=\frac{r}{n+1}$

Therefore it follows that $\frac{r}{n+1}=\mathbf{1}-\boldsymbol{e}^{-\left(\frac{x-\gamma}{\theta}\right)^{\beta}}$

Following Cohen and Whitten (1982), we set $r=1$. The first order statistic contains more information about $\gamma$ than any other order statistics and often more information than all the other order statistics combined.

When $r=1$

$$
\begin{gathered}
\frac{1}{n+1}=1-e^{-\left(\frac{x_{1}-\gamma}{\theta}\right)^{\beta}} \\
\frac{n}{n+1}=e^{-\left(\frac{x_{1}-\gamma}{\theta}\right)^{\beta}}
\end{gathered}
$$

taking logarithm of both sides gives; $\ln \left(\frac{n}{n+1}\right)=\ln \left(e^{-\left(\frac{x_{1}-\gamma}{\theta}\right)^{\beta}}\right)=\ln \left(\frac{n}{n+1}\right)=-\left(\frac{x_{1}-\gamma}{\theta}\right)^{\beta}$

$\therefore-\ln \left(\frac{n}{n+1}\right)=\left(\frac{x_{1}-\gamma}{\theta}\right)^{\beta}(2.25)$ 
Solving equations $3.25,3.26$ and 3.32 to eliminate $\theta$ and $\gamma$;

\section{Simulation and Comparison}

For the purpose of comparing the performance of the methods, the root mean square error (RMSE) was used in this work as a measure of accuracy of each method in estimating each of the three parameters. Also, the Euclidean norm was used to measure the total accuracy of each method in estimating the three parameters.

The RMSE of an estimator is calculated as;

$$
R M S E=\sqrt{\operatorname{Var}(x)+\text { bias }^{2}}
$$

The Euclidean norm, or Euclidean length or magnitude of a vector measures the length of the vector. It is given by;

$$
\|X\|=\sqrt{x_{1}^{2}+x_{2}^{2}+\cdots+x_{n}^{2}}
$$

For each method, a vector was formed using the root mean square error of the estimates of the three parameters, and then the distance will be calculated. Intuitively, since the vectors have errors (RMSE) as elements, it follows that the method that has a vector with shorter length is better than a method with a lengthier vector.

The values of the shape parameter for simulation were chosen based on the three regions of the bath-tub curve (as in reliability testing).

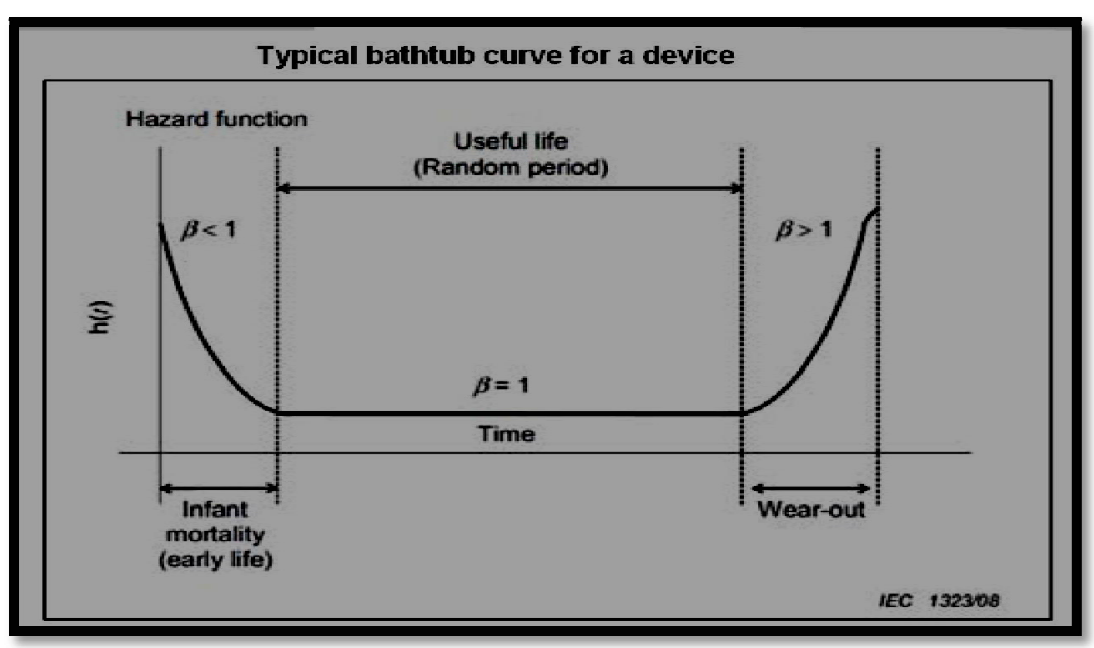

Figure 1: Bath-tub Curve

Source: www.reliawiki.com

Also, it is known that data gotten from reliability testing are always positively skewed. The Weibull distribution is positively skewed when $0.5<\beta<2$.6. Based on these, we chose $0.5,1,1.5$ and 2.5 as true values for $\beta$ to represent the three regions of the bath-tub curve $(\beta<1, \beta=1$ and $\beta>1$ respectively $)$. The scale and location parameters can vary depending on its unit of measurement, and since they are scaling parameters, we don't need to vary them. Therefore, we chose 100 and 10 as the values for the scale and location parameters respectively. We also varied the sample size (10, 20 and 50) to represent small, median and large sample sizes.4.0

\section{Results}

All computation such as generating random samples from the Weibull distribution, computation of estimates for the various methods were done using inbuilt and external packages in the R statistical software.

\begin{tabular}{|c|c|c|c|}
\hline True $\boldsymbol{\beta}$ & True $\boldsymbol{\theta}$ & True $\boldsymbol{\gamma}$ & Sample size n \\
\hline 0.5 & 100 & 10 & 10 \\
\hline 0.5 & 100 & 10 & 20 \\
\hline 0.5 & 100 & 10 & 50 \\
\hline 1 & 100 & 10 & 10 \\
\hline 1 & 100 & 10 & 20 \\
\hline 1 & 100 & 10 & 50 \\
\hline 1.5 & 100 & 10 & 10 \\
\hline 1.5 & 100 & 10 & 20 \\
\hline 1.5 & 100 & 10 & 50 \\
\hline 2.5 & 100 & 10 & 10 \\
\hline 2.5 & 100 & 10 & 20 \\
\hline 2.5 & 100 & 10 & 50 \\
\hline
\end{tabular}


Table 1: Categories of Data Simulation

\begin{tabular}{|c|c|c|c|}
\hline Methods & Parameters & BIAS & RMSE \\
\hline $\begin{array}{l}\text { MAHDI AND GUPTA } \\
\text { METHOD }\end{array}$ & $\begin{array}{l}\beta \\
\theta \\
\gamma\end{array}$ & $\begin{array}{c}0.1327512 \\
18.42228 \\
-2.163484 \\
\end{array}$ & $\begin{array}{l}0.1626008 \\
71.90500 \\
5.309800\end{array}$ \\
\hline $\begin{array}{l}\text { METHOD OF } \\
\text { L-MOMENTS }\end{array}$ & $\begin{array}{l}\beta \\
\theta \\
\gamma\end{array}$ & $\begin{array}{l}-0.023047 \\
-25.74027 \\
1.855000 \\
\end{array}$ & $\begin{array}{l}0.3765046 \\
74.389000 \\
10.155000 \\
\end{array}$ \\
\hline METHOD OF MOMENTS & $\begin{array}{l}\beta \\
\theta \\
\gamma\end{array}$ & $\begin{array}{l}0.215852 \\
-98.28613 \\
-7.527209 \\
\end{array}$ & $\begin{array}{c}0.2498848 \\
98.28782 \\
7.537441 \\
\end{array}$ \\
\hline PERCENTILE METHOD & $\begin{array}{l}\beta \\
\theta \\
\gamma\end{array}$ & $\begin{array}{c}-1.389274 \\
54.71819 \\
1.967292 \\
\end{array}$ & $\begin{array}{c}1.413455 \\
104.595 \\
4.552863 \\
\end{array}$ \\
\hline $\begin{array}{l}\text { MAXIMUM PRODUCT OF } \\
\text { SPACING }\end{array}$ & $\begin{array}{l}\beta \\
\theta \\
\gamma\end{array}$ & $\begin{array}{c}-0.0267443 \\
40.2032 \\
0.8197365\end{array}$ & $\begin{array}{c}0.1834215 \\
117.7352 \\
5.394052\end{array}$ \\
\hline $\begin{array}{l}\text { MODIFIED MOMENTS } \\
\text { METHOD }\end{array}$ & $\begin{array}{l}\beta \\
\theta \\
\gamma\end{array}$ & $\begin{array}{c}0.2443309 \\
54.03477 \\
-3.619115 \\
\end{array}$ & $\begin{array}{l}0.414332 \\
126.5229 \\
7.603042\end{array}$ \\
\hline $\begin{array}{l}\text { GODA'S POLYNOMIAL } \\
\text { METHOD }\end{array}$ & $\begin{array}{l}\beta \\
\theta \\
\gamma\end{array}$ & $\begin{array}{r}0.472447 \\
217.4522 \\
-6.567682 \\
\end{array}$ & $\begin{array}{l}1.084414 \\
796.6967 \\
9.461734 \\
\end{array}$ \\
\hline $\begin{array}{l}\text { MAXIMUM LIKELIHOOD } \\
\text { METHOD }\end{array}$ & $\begin{array}{l}\beta \\
\theta \\
\gamma\end{array}$ & NON-CONVERGENCE & NON-CONVERGENCE \\
\hline
\end{tabular}

Table 2: Results for Sample Size $=10$, Beta $=0.51$

\begin{tabular}{|c|c|c|c|}
\hline Methods & Parameters & BIAS & RMSE \\
\hline \multirow{2}{*}{$\begin{array}{c}\text { MAHDI AND GUPTA } \\
\text { METHOD }\end{array}$} & $\beta$ & -0.00234304 & 52.17188928 \\
& $\theta$ & -11.15802 & 1.247136 \\
\hline METHOD OF & $\beta$ & 0.4591412 & 0.2091123 \\
L-MOMENTS & $\theta$ & 0.06593231 & 74.67806 \\
& $\gamma$ & 11.60552 & 7.946221 \\
\hline
\end{tabular}




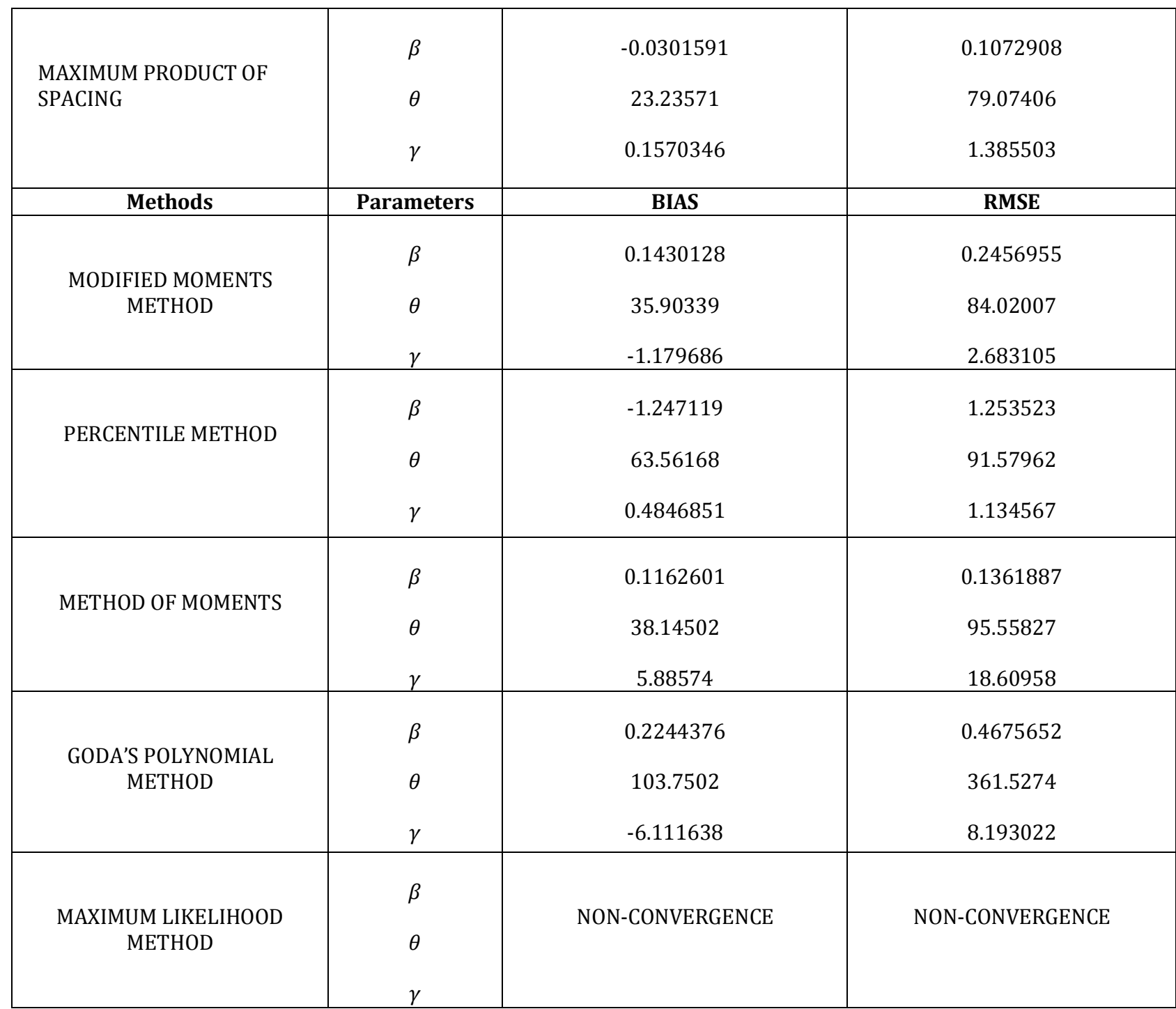

Table 3: Results for Sample Size $=20$ and Beta $=0.5$

\begin{tabular}{|c|c|c|c|}
\hline Methods & Parameters & BIAS & RMSE \\
\hline $\begin{array}{l}\text { MAHDI AND GUPTA } \\
\text { METHOD }\end{array}$ & $\begin{array}{l}\beta \\
\theta \\
\gamma\end{array}$ & $\begin{array}{c}4.37837 \mathrm{e}-05 \\
-5.792638 \\
0.06195151 \\
\end{array}$ & $\begin{array}{c}0.07617271 \\
31.03455 \\
0.1941643 \\
\end{array}$ \\
\hline $\begin{array}{l}\text { MAXIMUM PRODUCT OF } \\
\text { SPACING }\end{array}$ & $\begin{array}{l}\beta \\
\theta \\
\gamma\end{array}$ & $\begin{array}{c}-0.0200027 \\
11.67251 \\
0.0387571 \\
\end{array}$ & $\begin{array}{c}0.06271845 \\
38.5956 \\
0.1683332 \\
\end{array}$ \\
\hline $\begin{array}{l}\text { METHOD OF } \\
\text { L-MOMENTS }\end{array}$ & $\begin{array}{l}\beta \\
\theta \\
\gamma\end{array}$ & $\begin{array}{c}0.02439475 \\
2.624542 \\
-1.420584\end{array}$ & $\begin{array}{l}0.1095837 \\
39.62587 \\
5.558183\end{array}$ \\
\hline METHOD OF MOMENTS & $\begin{array}{l}\beta \\
\theta \\
\gamma\end{array}$ & $\begin{array}{c}0.05011312 \\
9.045346 \\
-6.94911 \\
\end{array}$ & $\begin{array}{c}0.07442058 \\
41.45837 \\
19.73336 \\
\end{array}$ \\
\hline $\begin{array}{l}\text { MODIFIED MOMENTS } \\
\text { METHOD }\end{array}$ & $\begin{array}{l}\beta \\
\theta\end{array}$ & $\begin{array}{c}0.07502497 \\
19.23583\end{array}$ & $\begin{array}{r}0.1424681 \\
46.64336\end{array}$ \\
\hline
\end{tabular}




\begin{tabular}{|c|c|c|c|}
\hline & $\gamma$ & -0.7588571 & 1.399621 \\
& & & \\
\hline Methods & Parameters & BIAS & RMSE \\
\hline PERCENTILE METHOD & $\beta$ & -1.196053 & 1.198189 \\
& $\theta$ & 63.76469 & 76.60829 \\
\hline GODA'S POLYNOMIAL & $\gamma$ & 0.07693967 & 0.173176 \\
\hline METHOD & $\theta$ & 0.08950027 & 0.1849431 \\
& $\gamma$ & 41.47789 & 124.5539 \\
\hline MAXIMUM LIKELIHOOD & $\beta$ & -4.831244 & 6.812224 \\
METHOD & $\theta$ & & NON-CONVERGENCE \\
& $\gamma$ & NON-CONVERGENCE & \\
\hline
\end{tabular}

Table 4: Results for Sample Size $=50$ and Beta $=0.5$

\begin{tabular}{|c|c|c|c|}
\hline Methods & Parameters & BIAS & RMSE \\
\hline $\begin{array}{l}\text { MAXIMUM PRODUCT OF } \\
\text { SPACING }\end{array}$ & $\begin{array}{l}\beta \\
\theta \\
\gamma \\
\end{array}$ & $\begin{array}{c}-0.0440639 \\
5.489691 \\
6.276413 \\
\end{array}$ & $\begin{array}{r}0.437876 \\
32.0976 \\
15.06475 \\
\end{array}$ \\
\hline $\begin{array}{l}\text { MAHDI AND GUPTA } \\
\text { METHOD }\end{array}$ & $\begin{array}{l}\beta \\
\theta \\
\gamma\end{array}$ & $\begin{array}{c}-0.1689997 \\
-23.16872 \\
10.29491 \\
\end{array}$ & $\begin{array}{r}0.2847648 \\
38.70501 \\
17.60728 \\
\end{array}$ \\
\hline PERCENTILE METHOD & $\begin{array}{l}\beta \\
\theta \\
\gamma\end{array}$ & $\begin{array}{l}-2.598237 \\
11.08241 \\
9.663415 \\
\end{array}$ & $\begin{array}{l}2.643818 \\
35.97132 \\
14.21158 \\
\end{array}$ \\
\hline METHOD OF MOMENTS & $\begin{array}{l}\beta \\
\theta \\
\gamma \\
\end{array}$ & $\begin{array}{r}0.3668697 \\
-31.32084 \\
24.46045 \\
\end{array}$ & $\begin{array}{l}0.448224 \\
42.67084 \\
29.18214 \\
\end{array}$ \\
\hline $\begin{array}{l}\text { MODIFIED MOMENTS } \\
\text { METHOD }\end{array}$ & $\begin{array}{l}\beta \\
\theta \\
\gamma\end{array}$ & $\begin{array}{c}0.825923 \\
17.4023 \\
-2.457843\end{array}$ & $\begin{array}{l}1.313565 \\
58.94141 \\
10.15621 \\
\end{array}$ \\
\hline $\begin{array}{l}\text { METHOD OF } \\
\text { L-MOMENTS }\end{array}$ & $\begin{array}{l}\beta \\
\theta \\
\gamma\end{array}$ & $\begin{array}{c}0.5012449 \\
25.06442 \\
0.3177051 \\
\end{array}$ & $\begin{array}{l}2.086022 \\
148.8132 \\
12.79854 \\
\end{array}$ \\
\hline $\begin{array}{l}\text { GODA'S POLYNOMIAL } \\
\text { METHOD }\end{array}$ & $\begin{array}{l}\beta \\
\theta \\
\gamma\end{array}$ & $\begin{array}{l}0.5120562 \\
35.00642 \\
7.849979\end{array}$ & $\begin{array}{l}1.977292 \\
151.9566 \\
27.45279 \\
\end{array}$ \\
\hline
\end{tabular}




\begin{tabular}{|c|c|c|c|}
\hline $\begin{array}{c}\text { MAXIMUM LIKELIHOOD } \\
\text { METHOD }\end{array}$ & $\beta$ & NON-CONVERGENCE & NON-CONVERGENCE \\
& $\gamma$ & & \\
\hline
\end{tabular}

Table 5: Results for Sample Size $=10$ and Beta=1

\begin{tabular}{|c|c|c|c|}
\hline Methods & Parameter & BIAS & RMSE \\
\hline $\begin{array}{l}\text { MAHDI AND GUPTA } \\
\text { METHOD }\end{array}$ & $\begin{array}{l}\beta \\
\theta \\
\gamma\end{array}$ & $\begin{array}{c}-0.08500607 \\
-11.91453 \\
4.938966 \\
\end{array}$ & $\begin{array}{l}0.1989304 \\
25.06324 \\
8.725661 \\
\end{array}$ \\
\hline PERCENTILE METHOD & $\begin{array}{l}\beta \\
\theta \\
\gamma\end{array}$ & $\begin{array}{l}-2.35998 \\
14.1715 \\
4.905211 \\
\end{array}$ & $\begin{array}{r}2.373444 \\
28.64229 \\
6.784815 \\
\end{array}$ \\
\hline $\begin{array}{l}\text { MODIFIED MOMENTS } \\
\text { METHOD }\end{array}$ & $\begin{array}{l}\beta \\
\theta \\
\gamma\end{array}$ & $\begin{array}{l}0.6613739 \\
5.554375 \\
-2.070715 \\
\end{array}$ & $\begin{array}{l}1.059958 \\
32.91854 \\
6.851777 \\
\end{array}$ \\
\hline $\begin{array}{l}\text { MAXIMUM PRODUCT OF } \\
\text { SPACING }\end{array}$ & $\begin{array}{l}\beta \\
\theta \\
\gamma\end{array}$ & $\begin{array}{c}-0.0161961 \\
4.065683 \\
1.754199 \\
\end{array}$ & $\begin{array}{r}0.437876 \\
32.0976 \\
15.06475 \\
\end{array}$ \\
\hline $\begin{array}{l}\text { METHOD OF } \\
\text { L-MOMENTS }\end{array}$ & $\begin{array}{l}\beta \\
\theta \\
\gamma\end{array}$ & $\begin{array}{r}0.1330612 \\
4.852756 \\
-0.1622821 \\
\end{array}$ & $\begin{array}{l}0.562391 \\
41.89465 \\
9.785603 \\
\end{array}$ \\
\hline $\begin{array}{c}\text { GODA'S POLYNOMIAL } \\
\text { METHOD }\end{array}$ & $\begin{array}{l}\beta \\
\theta \\
\gamma\end{array}$ & $\begin{array}{r}0.1305346 \\
4.717018 \\
-0.1522319 \\
\end{array}$ & $\begin{array}{l}0.5352034 \\
41.03389 \\
9.807257 \\
\end{array}$ \\
\hline METHOD OF MOMENTS & $\begin{array}{l}\beta \\
\theta \\
\gamma\end{array}$ & $\begin{array}{l}0.249682 \\
-44.0607 \\
31.21525 \\
\end{array}$ & $\begin{array}{l}0.2905533 \\
47.35216 \\
33.20622 \\
\end{array}$ \\
\hline $\begin{array}{l}\text { MAXIMUM LIKELIHOOD } \\
\text { METHOD }\end{array}$ & $\begin{array}{l}\beta \\
\theta \\
\gamma\end{array}$ & NON-CONVERGENCE & NON-CONVERGENCE \\
\hline
\end{tabular}

Table 6: Results for Sample Size $=20$ and Beta=1

\begin{tabular}{|c|c|c|c|}
\hline Methods & Parameters & BIAS & RMSE \\
\hline & $\beta$ & -0.0327187 & 0.4812885 \\
MAHDI AND GUPTA & $\theta$ & -4.030083 & 15.42769 \\
METHOD & $\gamma$ & 1.97096 & 3.39117 \\
\hline
\end{tabular}




\begin{tabular}{|c|c|c|c|}
\hline $\begin{array}{l}\text { MAXIMUM PRODUCT OF } \\
\text { SPACING }\end{array}$ & $\begin{array}{l}\beta \\
\theta \\
\gamma\end{array}$ & $\begin{array}{l}-0.030265 \\
1.979195 \\
0.0293641 \\
\end{array}$ & $\begin{array}{c}0.1357624 \\
16.39277 \\
2.363727 \\
\end{array}$ \\
\hline $\begin{array}{l}\text { MODIFIED MOMENTS } \\
\text { METHOD }\end{array}$ & $\begin{array}{l}\beta \\
\theta \\
\gamma\end{array}$ & $\begin{array}{l}0.1015814 \\
8.168283 \\
-4.894601\end{array}$ & $\begin{array}{c}0.2163439 \\
20.57716 \\
7.771806\end{array}$ \\
\hline Methods & Parameters & BIAS & RMSE \\
\hline $\begin{array}{l}\text { METHOD OF } \\
\text { L-MOMENTS }\end{array}$ & $\begin{array}{l}\beta \\
\theta \\
\gamma\end{array}$ & $\begin{array}{c}0.03443095 \\
1.450009 \\
-0.4822946\end{array}$ & $\begin{array}{c}0.2054361 \\
21.47622 \\
7.096058 \\
\end{array}$ \\
\hline PERCENTILE METHOD & $\begin{array}{l}\beta \\
\theta \\
\gamma\end{array}$ & $\begin{array}{l}-2.236591 \\
16.11249 \\
1.975506 \\
\end{array}$ & $\begin{array}{r}2.24113 \\
24.47369 \\
2.743128 \\
\end{array}$ \\
\hline $\begin{array}{l}\text { GODA'S POLYNOMIAL } \\
\text { METHOD }\end{array}$ & $\begin{array}{l}\beta \\
\theta \\
\gamma\end{array}$ & $\begin{array}{l}0.1305346 \\
10.05517 \\
2.422828\end{array}$ & $\begin{array}{c}0.5352034 \\
51.11268 \\
13.46079\end{array}$ \\
\hline METHOD OF MOMENTS & $\begin{array}{l}\beta \\
\theta \\
\gamma\end{array}$ & $\begin{array}{l}0.1735489 \\
-52.11863 \\
36.78098\end{array}$ & $\begin{array}{l}0.1882376 \\
53.15337 \\
37.51275\end{array}$ \\
\hline $\begin{array}{l}\text { MAXIMUM LIKELIHOOD } \\
\text { METHOD }\end{array}$ & $\begin{array}{l}\beta \\
\theta \\
\gamma\end{array}$ & NON-CONVERGENCE & NON-CONVERGENCE \\
\hline
\end{tabular}

Table 7: Results for Sample Size $=50$ and Beta $=1$

\begin{tabular}{|c|c|c|c|}
\hline Methods & Parameters & Bias & Rmse \\
\hline \multirow{2}{*}{ PERCENTILE METHOD } & $\beta$ & -3.796833 & 24.855535 \\
& $\theta$ & 6.718831 & 23.12697 \\
\hline \multirow{2}{*}{$\begin{array}{c}\text { MAHDI AND GUPTA } \\
\text { METHOD }\end{array}$} & $\beta$ & 19.17645 & 0.5364848 \\
& $\theta$ & -0.4584609 & 37.86138 \\
\hline GODA'S POLYNOMIAL & $\gamma$ & -31.26084 & 23.54054 \\
\hline METHOD & $\beta$ & 19.34675 & 3.3298 \\
\hline
\end{tabular}




\begin{tabular}{|c|c|c|c|}
\hline $\begin{array}{c}\text { MAXIMUM LIKELIHOOD } \\
\text { METHOD }\end{array}$ & $\beta$ & 0.7095411 & 3.965586 \\
& $\gamma$ & 18.15194 & 167.2714 \\
\hline $\begin{array}{c}\text { MAXIMUM PRODUCT OF } \\
\text { SPACING }\end{array}$ & $\beta$ & 41.62018 & 164.8175 \\
\hline & $\gamma$ & 0.5571118 & 8.497256 \\
& $\gamma$ & 35.23447 & 203.1478 \\
\hline Methods & Parameters & 43.43592 & 157.7633 \\
\hline METHOD OF MOMENTS & $\beta$ & Bias & Rmse \\
\hline MODIFIED MOMENTS & $\theta$ & NO ROOT & NO ROOT \\
\hline METHOD & $\gamma$ & & NO ROOT \\
\hline METHOD OF & $\gamma$ & NO ROOT & \\
\hline & $\gamma$ & & NO ROOT \\
\hline & $\gamma$ & & \\
\hline
\end{tabular}

Table 8: Results for Sample Size $=10$ and Beta $=1.5$

\begin{tabular}{|c|c|c|c|}
\hline Methods & Parameters & BIAS & RMSE \\
\hline PERCENTILE METHOD & $\begin{array}{l}\beta \\
\theta \\
\gamma\end{array}$ & $\begin{array}{c}-3.469112 \\
8.56358 \\
12.17397 \\
\end{array}$ & $\begin{array}{r}3.49189 \\
19.08257 \\
14.49892 \\
\end{array}$ \\
\hline $\begin{array}{l}\text { MAHDI AND GUPTA } \\
\text { METHOD }\end{array}$ & $\begin{array}{l}\beta \\
\theta \\
\gamma\end{array}$ & $\begin{array}{c}-0.2805753 \\
-17.20969 \\
12.10774 \\
\end{array}$ & $\begin{array}{l}0.3622391 \\
23.52643 \\
14.44512 \\
\end{array}$ \\
\hline $\begin{array}{l}\text { MAXIMUM PRODUCT OF } \\
\text { SPACING }\end{array}$ & $\begin{array}{l}\beta \\
\theta \\
\gamma\end{array}$ & $\begin{array}{l}0.1232299 \\
10.53943 \\
9.415137 \\
\end{array}$ & $\begin{array}{c}8.546578 \\
119.491 \\
57.81012 \\
\end{array}$ \\
\hline $\begin{array}{l}\text { MAXIMUM LIKELIHOOD } \\
\text { METHOD }\end{array}$ & $\begin{array}{l}\beta \\
\theta \\
\gamma\end{array}$ & $\begin{array}{c}0.0107799 \\
-5.359939 \\
10.65083\end{array}$ & $\begin{array}{l}0.979813 \\
36.87105 \\
27.64767\end{array}$ \\
\hline $\begin{array}{l}\text { MODIFIED MOMENTS } \\
\text { METHOD }\end{array}$ & $\begin{array}{l}\beta \\
\theta \\
\gamma\end{array}$ & $\begin{array}{c}0.4452731 \\
-40.0008 \\
37.4892 \\
\end{array}$ & $\begin{array}{r}2.433209 \\
50.0004 \\
38.0003 \\
\end{array}$ \\
\hline
\end{tabular}




\begin{tabular}{|c|c|c|c|}
\hline & $\beta$ & 0.3061825 & 1.238395 \\
$\begin{array}{c}\text { METHOD OF } \\
\text { L-MOMENTS }\end{array}$ & $\theta$ & 12.37453 & 52.99993 \\
& $\gamma$ & 0.5281383 & 11.67473 \\
\hline METHOD OF MOMENTS & $\beta$ & -0.6032349 & 0.6448244 \\
& $\theta$ & -49.3039 & 53.42191 \\
\hline GODA'S POLYNOMIAL & $\gamma$ & 36.7787 & 40.31892 \\
\hline METHOD & $\theta$ & 0.2732907 & 0.9382057 \\
\hline
\end{tabular}

Table 9: Results for Sample Size $=20$ and Beta $=1.5$

\begin{tabular}{|c|c|c|c|}
\hline Methods & Parameters & BIAS & RMSE \\
\hline $\begin{array}{l}\text { MAXIMUM LIKELIHOOD } \\
\text { METHOD }\end{array}$ & $\begin{array}{l}\beta \\
\theta \\
\gamma\end{array}$ & $\begin{array}{c}-0.05796005 \\
-5.63222 \\
3.972861 \\
\end{array}$ & $\begin{array}{r}0.2651848 \\
14.09852 \\
6.807028 \\
\end{array}$ \\
\hline $\begin{array}{l}\text { MAHDI AND GUPTA } \\
\text { METHOD }\end{array}$ & $\begin{array}{l}\beta \\
\theta \\
\gamma\end{array}$ & $\begin{array}{c}-0.1574609 \\
-9.816728 \\
6.803893 \\
\end{array}$ & $\begin{array}{c}0.2237386 \\
14.2841 \\
8.214172 \\
\end{array}$ \\
\hline PERCENTILE METHOD & $\begin{array}{l}\beta \\
\theta \\
\gamma\end{array}$ & $\begin{array}{c}-3.309581 \\
10.35368 \\
6.845279 \\
\end{array}$ & $\begin{array}{c}3.316965 \\
15.80495 \\
8.24641 \\
\end{array}$ \\
\hline $\begin{array}{l}\text { GODA'S POLYNOMIAL } \\
\text { METHOD }\end{array}$ & $\begin{array}{l}\beta \\
\theta \\
\gamma\end{array}$ & $\begin{array}{c}0.05491001 \\
1.607711 \\
0.2095117 \\
\end{array}$ & $\begin{array}{l}0.366442 \\
20.50919 \\
8.785405 \\
\end{array}$ \\
\hline $\begin{array}{l}\text { METHOD OF } \\
\text { L-MOMENTS }\end{array}$ & $\begin{array}{l}\beta \\
\theta \\
\gamma\end{array}$ & $\begin{array}{c}0.05359229 \\
1.515607 \\
0.2912337 \\
\end{array}$ & $\begin{array}{r}0.3683909 \\
20.59285 \\
8.828512 \\
\end{array}$ \\
\hline METHOD OF MOMENTS & $\begin{array}{l}\beta \\
\theta \\
\gamma\end{array}$ & $\begin{array}{c}0.4713041 \\
25.24634 \\
-7.237766 \\
\end{array}$ & $\begin{array}{r}1.045212 \\
53.46118 \\
9.045932 \\
\end{array}$ \\
\hline $\begin{array}{l}\text { MODIFIED MOMENTS } \\
\text { METHOD }\end{array}$ & $\begin{array}{l}\beta \\
\theta \\
\gamma\end{array}$ & $\begin{array}{c}-0.6965429 \\
-57.33639 \\
41.39441 \\
\end{array}$ & $\begin{array}{c}0.7087268 \\
58.30003 \\
42.10247 \\
\end{array}$ \\
\hline $\begin{array}{l}\text { MAXIMUM PRODUCT OF } \\
\text { SPACING }\end{array}$ & $\begin{array}{l}\beta \\
\theta \\
\gamma\end{array}$ & $\begin{array}{c}-0.03404346 \\
1.349955 \\
0.1827269\end{array}$ & $\begin{array}{l}8.538009 \\
92.45336 \\
6.001584 \\
\end{array}$ \\
\hline
\end{tabular}

Table 10: Results for Sample Size $=50$ and Beta $=1.5$ 


\begin{tabular}{|c|c|c|c|}
\hline Methods & Parameters & BIAS & RMSE \\
\hline PERCENTILE METHOD & $\begin{array}{l}\beta \\
\theta \\
\gamma\end{array}$ & $\begin{array}{l}-5.188339 \\
5.860043 \\
35.59741 \\
\end{array}$ & $\begin{array}{l}5.300268 \\
15.87704 \\
38.51351\end{array}$ \\
\hline $\begin{array}{l}\text { MAHDI AND GUPTA } \\
\text { METHOD }\end{array}$ & $\begin{array}{l}\beta \\
\theta \\
\gamma\end{array}$ & $\begin{array}{l}-1.231671 \\
-43.98572 \\
35.7258 \\
\end{array}$ & $\begin{array}{l}1.283226 \\
41.46263 \\
38.80891 \\
\end{array}$ \\
\hline $\begin{array}{l}\text { MAXIMUM } \quad \text { PRODUCT } \quad \text { OF } \\
\text { SPACING }\end{array}$ & $\begin{array}{l}\beta \\
\theta \\
\gamma\end{array}$ & $\begin{array}{l}1.08509 \\
-4.97473 \\
25.40446\end{array}$ & $\begin{array}{l}1.224916 \\
44.38471 \\
33.44971\end{array}$ \\
\hline Methods & Parameters & BIAS & RMSE \\
\hline MAXIMUM LIKELIHOOD METHOD & $\begin{array}{l}\beta \\
\theta \\
\gamma\end{array}$ & $\begin{array}{l}0.935712 \\
11.47466 \\
65.26447 \\
\end{array}$ & $\begin{array}{l}5.151017 \\
140.8101 \\
153.7486 \\
\end{array}$ \\
\hline $\begin{array}{l}\text { GODA'S POLYNOMIAL } \\
\text { METHOD }\end{array}$ & $\begin{array}{l}\beta \\
\theta \\
\gamma\end{array}$ & $\begin{array}{l}2.992037 \\
48.19675 \\
14.89093\end{array}$ & $\begin{array}{l}7.289067 \\
255.5098 \\
29.15153\end{array}$ \\
\hline METHOD OF MOMENTS & $\begin{array}{l}\beta \\
\theta \\
\gamma\end{array}$ & NO ROOT & NO ROOT \\
\hline $\begin{array}{l}\text { METHOD OF } \\
\text { L-MOMENTS }\end{array}$ & $\begin{array}{l}\beta \\
\theta \\
\gamma\end{array}$ & NO ROOT & NO ROOT \\
\hline
\end{tabular}

Table 11: Results for Sample Size $=10$ and Beta $=2.5$

\begin{tabular}{|c|c|c|c|}
\hline Methods & Parameters & BIAS & RMSE \\
\hline PERCENTILE METHOD & $\begin{array}{l}\beta \\
\theta \\
\gamma\end{array}$ & $\begin{array}{l}-4.757424 \\
7.411526 \\
27.00638 \\
\end{array}$ & $\begin{array}{l}4.801904 \\
13.24821 \\
29.26563 \\
\end{array}$ \\
\hline $\begin{array}{l}\text { MAHDI AND GUPTA } \\
\text { METHOD }\end{array}$ & $\begin{array}{l}\beta \\
\theta \\
\gamma\end{array}$ & $\begin{array}{c}-0.9306541 \\
-32.20311 \\
27.42744 \\
\end{array}$ & $\begin{array}{r}0.9873057 \\
35.10124 \\
29.91266 \\
\end{array}$ \\
\hline METHOD OF MOMENTS & $\begin{array}{l}\beta \\
\theta \\
\gamma\end{array}$ & $\begin{array}{l}-1.367543 \\
-56.18469 \\
45.68911 \\
\end{array}$ & $\begin{array}{r}1.439514 \\
59.88441 \\
48.35342 \\
\end{array}$ \\
\hline $\begin{array}{l}\text { GODA'S POLYNOMIAL } \\
\text { METHOD }\end{array}$ & $\begin{array}{l}\beta \\
\theta \\
\gamma\end{array}$ & $\begin{array}{c}0.6378122 \\
-73.63471 \\
65.34878 \\
\end{array}$ & $\begin{array}{l}3.358856 \\
75.82363 \\
67.54127 \\
\end{array}$ \\
\hline MAXIMUM LIKELIHOOD METHOD & $\begin{array}{l}\beta \\
\theta\end{array}$ & $\begin{array}{c}0.5162815 \\
5.064054\end{array}$ & $\begin{array}{l}3.919403 \\
101.0869\end{array}$ \\
\hline
\end{tabular}




\begin{tabular}{|c|c|c|c|}
\hline & $\gamma$ & 32.27247 & 107 \\
\hline $\begin{array}{c}\text { MAXIMUM PRODUCT OF } \\
\text { SPACING }\end{array}$ & $\beta$ & 0.9545239 & 5.320103 \\
& $\theta$ & 36.00158 & 149.4277 \\
\hline METHOD OF & $\gamma$ & 46.37472 & NO ROOT \\
\hline L-MOMENTS & $\theta$ & NO ROOT & NO ROOT \\
\hline MODIFIED MOMENTS & $\gamma$ & & NO ROOT \\
\hline METHOD & $\theta$ & & \\
\hline
\end{tabular}

Table 12: Results for Sample Size $=20$ and Beta $=2.5$

\begin{tabular}{|c|c|c|c|}
\hline Methods & Parameters & BIAS & RMSE \\
\hline PERCENTILE METHOD & $\begin{array}{l}\beta \\
\theta \\
\gamma\end{array}$ & $\begin{array}{r}-4.483661 \\
8.235697 \\
18.81099 \\
\end{array}$ & $\begin{array}{r}4.501284 \\
11.01037 \\
20.41387 \\
\end{array}$ \\
\hline $\begin{array}{c}\text { MAHDI AND GUPTA } \\
\text { METHOD }\end{array}$ & $\begin{array}{l}\beta \\
\theta \\
\gamma\end{array}$ & $\begin{array}{c}-0.6004832 \\
-21.00493 \\
1.970963 \\
\end{array}$ & $\begin{array}{l}0.655485 \\
23.08954 \\
2.759584 \\
\end{array}$ \\
\hline $\begin{array}{l}\text { MAXIMUM LIKELIHOOD } \\
\text { METHOD }\end{array}$ & $\begin{array}{l}\beta \\
\theta \\
\gamma\end{array}$ & $\begin{array}{r}0.03464351 \\
-3.083195 \\
8.928148 \\
\end{array}$ & $\begin{array}{c}0.8526618 \\
23.71099 \\
17.58165 \\
\end{array}$ \\
\hline $\begin{array}{l}\text { MAXIMUM PRODUCT OF } \\
\text { SPACING }\end{array}$ & $\begin{array}{l}\beta \\
\theta \\
\gamma\end{array}$ & $\begin{array}{c}0.1474586 \\
7.695812 \\
6.584551 \\
\end{array}$ & $\begin{array}{c}0.8400696 \\
26.60051 \\
17.83054 \\
\end{array}$ \\
\hline $\begin{array}{l}\text { GODA'S POLYNOMIAL } \\
\text { METHOD }\end{array}$ & $\begin{array}{l}\beta \\
\theta \\
\gamma\end{array}$ & $\begin{array}{l}0.122791 \\
6.149524 \\
2.211636 \\
\end{array}$ & $\begin{array}{r}0.9142861 \\
29.39152 \\
12.77273 \\
\end{array}$ \\
\hline $\begin{array}{l}\text { METHOD OF } \\
\text { L-MOMENTS }\end{array}$ & $\begin{array}{l}\beta \\
\theta \\
\gamma\end{array}$ & $\begin{array}{c}0.2517798 \\
6.620358 \\
1.813612 \\
\end{array}$ & $\begin{array}{l}0.951136 \\
29.60712 \\
12.63197 \\
\end{array}$ \\
\hline METHOD OF MOMENTS & $\begin{array}{l}\beta \\
\theta \\
\gamma\end{array}$ & $\begin{array}{r}-1.370495 \\
-50.18469 \\
45.68911 \\
\end{array}$ & $\begin{array}{l}1.409083 \\
52.88441 \\
41.35342 \\
\end{array}$ \\
\hline $\begin{array}{l}\text { METHOD OF } \\
\text { L-MOMENTS }\end{array}$ & $\begin{array}{l}\beta \\
\theta \\
\gamma\end{array}$ & $\begin{array}{c}0.2517798 \\
6.620358 \\
1.813612 \\
\end{array}$ & $\begin{array}{l}0.951136 \\
29.60712 \\
12.63197 \\
\end{array}$ \\
\hline
\end{tabular}


Table 13: Results for Sample Size $=50$ and Beta $=2.5$

\section{Findings}

From the results of the simulation experiments, the Teimouri and Gupta method is the best method of estimating the parameters of the three-parameter Weibull distribution. On the average, it produces very good estimates for small, medium and large sample sizes and across the different beta values.

The maximum product of spacing and the percentile method follow behind the Teimouri and Gupta method. Though the maximum product of spacing did not do well when $\beta=2.5$.

The method of moment and the modified method of moments performed relatively below average and even produced too many 'no root' situations when $\beta=2.5$. The method of L-moment also did not perform relatively well across the simulated samples. The polynomial didn't do well too.

No roots mean there were too many no situations were no root was found using a particular method. Nonconvergence means that there were too many situations were a particular method did not converge.

\subsection{Application to Real Data}

Two real life data sets are presented and the methods of estimation are used to find estimates for each data set.

Bearing cage fracture times (hrs) $\quad 230,334,423,990,1009,1510$

Table 14: Data of Time to Failure of Airplane Glass

Source: Abernethy Et Al Weibull Handbook (AFWAL-TR-83-2079) Pg. 43

failure times locomotive controls (miles)
$225,375,460,485,515,530,545,575,665,680,695,765,770,785,800$, $815,820,830,840,915,935,1025,1070,1085,1125,1135,1160,1170$,

$1185,1190,1200,1225,1230,1275,1310,1325,1340$

Table 15: Data of Failure Times Locomotive Controls

Source: Hahn G.J. and Shapiro S.S (1967); Statistical Models in Engineering

\begin{tabular}{|c|c|c|c|}
\hline \multicolumn{3}{|c|}{ ESTIMATES } & $\gamma$ \\
\hline Teimouri and Gupta Method & $\beta$ & $\theta$ & 229.8333 \\
\hline Method of L-moments & 0.6933112 & 406.6268 & 747.9875 \\
\hline Modified Method of Moment & 1.519542 & 907.8518 & 191.0501 \\
\hline Maximum Product of Spacing & 1.117227 & 581.4921 & 146.6762 \\
\hline Goda's Polynomial & 0.8890775 & 688.6378 & 747.9639 \\
\hline Method of Moments & 1.522161 & 909.2661 & - \\
\hline Percentile Method & 1.971364 & - & 230.052 \\
\hline Maximum Likelihood Method & 0.5938066 & 442.4246550 & 230.000 \\
\hline
\end{tabular}

Table 16 Result for Bearing Cage Fracture Times Data (Sample Size =6)

\begin{tabular}{|c|c|c|c|}
\hline \multicolumn{3}{|c|}{ ESTIMATES } \\
\hline & $\boldsymbol{\beta}$ & $\boldsymbol{\theta}$ & $\boldsymbol{\gamma}$ \\
\hline Teimouri And Gupta Method & 2.240967 & 755.2693 & 887.8253 \\
\hline Method Of L-Moments & 6.537696 & 1872.262 & No result \\
\hline Modified Method of Moment & No result & No result & 2026.893 \\
\hline Maximum Product of Spacing & 10.81984 & 3056.769 & 888.5162 \\
\hline Goda's Polynomial & 5.832769 & 1691.457 & 225.54 \\
\hline Percentile Method & 3.367362 & 893.6662 & - \\
\hline
\end{tabular}

Table 17: Result for Failure Times of Locomotive Controls (Sample Size = 37)

\subsection{Conclusion}

From the above findings, the Teimouri and Gupta method is the best method for estimating the parameters of the three-parameter Weibull distribution when applied to reliability data (i.e. when the distribution is positively skewed). Sample size does not affect the choice of method, though the performances of the methods always improved with sample size.

\section{References}


i. Cheng, R. C. H. and Amin, N. A. K. (1979). Maximum Product of Spacing Estimation With Application To The LogNormal Distribution, University of Wales IST, Methat Report 79.

ii. Cheng, R. C. H. and Amin, N. A. K. (1983). Estimating Parameters In Continuous Univariate Distributions With A Shifted Origin, Journal of the Royal Statistical Society, Series B (Methodological) 45(3), 394-403.

iii. Cohen, A. C and Whitten, B (1982). Modified Maximum Likelihood And Modified Moment

iv. Estimators For The Three-Parameter Weibull Distribution, Communications in Statistics

v. Theory and Methods 11.

vi. Dubey S.Y.D (1967). Normal and weibull distributions, naval research logistics quarterly, 14(1), 69-79 dio:10.1004nav.3800140107.

vii. Goda, Y., Kudaka, M and Kawai, H. (2010). Incorporation Of Weibull Distribution In L-

viii. Moments Method For Regional Frequency Analysis Of Peaks-Over-Threshold Wave Height

ix. Proceedings of the International Conference on Coastal Engineering,

x. http://journals.tdl.org/ICCE/article/viewFile/1154/pdf_42.

xi. $\quad$ Lloyd, N. S. (1967). Weibull Probability Paper, Industrial Quality Control 23, 452-453.

xii. Teimouri M. and Gupta A.K (2013). On the three-parameter weibull distribution shape parameter estimation, journal of data science 11, 403-414. 